\title{
ON A THEOREM OF DVORETSKY, WALD, AND WOLFOWITZ CONCERNING LIAPOUNOV MEASURES
}

\author{
by D. A. EDWARDS
}

(Received 25 January, 1986)

1. Introduction. Let $\Omega$ be a non-empty set, $\mathscr{F}$ a Boolean $\sigma$-algebra of subsets of $\Omega$, $k$ a natural number, and let $m: \mathscr{F} \rightarrow \mathbb{R}^{k}$ be a non-atomic vector measure. Then, by the celebrated theorem of Liapounov [11], the range $m[\mathscr{F}]=\{m(A): A \in \mathscr{F}\}$ of $m$ is a compact convex subset of $\mathbb{R}^{k}$. This theorem has been generalized in a number of ways. For example Kingman and Robertson [8] and Knowles [9] have shown that, under appropriate conditions, results in the same spirit can be proved for measures taking their values in infinite-dimensional vector spaces. Another type of generalization was obtained by Dvoretsky, Wald and Wolfowitz [6,7]. What they do is to take $m$ as above together with a natural number $n \geq 1$. They then consider the set $K_{n}$ of all vectors

$$
\left(m\left(A_{1}\right), m\left(A_{2}\right), \ldots, m\left(A_{n}\right)\right) \in \mathbb{R}^{n k},
$$

where $\left(A_{1}, A_{2}, \ldots, A_{n}\right)$ is an ordered $\mathscr{F}$-measurable partition of $\Omega$ (i.e. a partition whose terms $A_{r}$ all belong to $\mathscr{F}$ ). They prove in [6] that $K_{n}$ is a compact convex subset of $\mathbb{R}^{n k}$, and moreover that $K_{n}$ is equal to the set of all vectors of the form

$$
\left(\int \phi_{1} d m, \int \phi_{2} d m, \ldots, \int \phi_{n} d m\right),
$$

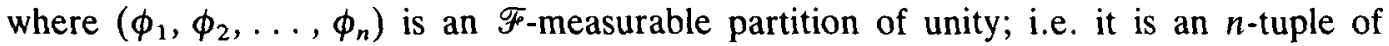
non-negative $\mathscr{F}$-measurable real functions $\phi_{r}$ on $\Omega$ such that

$$
\sum_{r=1}^{n} \phi_{r}(\omega)=1 \quad(\omega \in \Omega) \text {. }
$$

Liapounov's theorem can be obtained as a corollary of this result by taking $n=2$.

The present paper has two main objectives.

(1) To extend the Dvoretsky-Wald-Wolfowitz theorem to appropriate vector measures with infinite-dimensional range space.

(2) To develop the methods used for (1) to obtain results in the same vein for countably infinite $\mathscr{F}$-measurable partitions of $\Omega$.

Both objectives are reached with the help of techniques and ideas due to Lindenstrauss [10], Kingman and Robertson [8], and Knowles [9], and we obtain incidentally a somewhat simpler proof of the original theorem of Dvoretsky, Wald and Wolfowitz [6].

It is convenient to explain now some of the terminology and notation to be used. We can then state precisely some of the main results to be proved.

We shall denote by $X$ a real Banach space, and by $m$ we shall in future always mean a (countably additive) measure $m: \mathscr{F} \rightarrow X$. (A good general reference on vector measures

Glasgow Math. J. 29 (1987) 205-220. 
is [3]). We recall that, by a theorem of Bartle, Dunford and Schwartz [2] there exists a probability measure $\mu: \mathscr{F} \rightarrow \mathbb{B}$ such that, for all $A \in \mathscr{F}, \mu(A)=0$ if and only if $m(A \cap B)=0$ for all $B \in \mathscr{F}$. Any such $\mu$ is unique up to equivalence of measures. Given $m: \mathscr{F} \rightarrow X$ we choose such a $\mu$ once and for all and call it a control measure for $m$. If $\phi \in L^{\infty}(\mu)$ we can construct

$$
m(\phi)=\int \phi d m
$$

either as a weak integral or as in [2]. Both methods yield the same $m(\phi) \in X$ and we have

$$
x^{*}(m(\phi))=\int \phi d\left(x^{*} \circ m\right)
$$

for all $\phi \in L^{\infty}(\mu), x^{*} \in X^{*}$. If $\phi=0$ a.e. $(\mu)$, then $m(\phi)=0$. Since $x^{*} \circ m$ has an $L^{1}$ density with respect to $\mu$ we see at once that the map

$$
L^{\infty}(\mu) \ni \phi \mapsto m(\phi) \in X
$$

is continuous with respect to the topologies $\sigma\left(L^{\infty}(\mu), L^{1}(\mu)\right)$ and $\sigma\left(X, X^{*}\right)$.

Now let

$$
N=\left\{\phi \in L^{\infty}(\mu): m(\phi)=0\right\}
$$

and for each $S \in \mathscr{F}$ let

$$
N(S)=\{\phi \in N: \phi=0 \text { a.e. }(\mu) \text { in } \Omega \backslash S\} .
$$

We call $m$ a Liapounov measure if $N(S)$ is non-trivial for all $S \in \mathscr{F}$ such that $\mu(S)>0$. In this Banach-space setting the Knowles extension [9] of Liapounov's theorem states that $m$ is a Liapounov measure if and only if for each $A \in \mathscr{F}$ the measure $m_{A}$ defined by $m_{A}(B)=m(A \cap B)(B \in \mathscr{F})$ has as range $m_{A}[\mathscr{F}]$ a weakly compact convex subset of $X$. This does extend the Liapounov theorem, because it is known that when $X$ is finite-dimensional $m$ is Liapounov if and only if it is non-atomic [3]. When $X$ is infinite-dimensional non-atomicity of $m$ is necessary but not sufficient for it to be Liapounov [3].

Now agree that two sets $A, B \in \mathscr{F}$ are almost disjoint if $\mu(A \cap B)=\phi$, and let $\mathscr{F}_{n}(\mu)$ be the set of all

$$
\mathbf{A}=\left(A_{1}, A_{2}, \ldots, A_{n}\right) \in \mathscr{F}^{n}
$$

such that the $A_{r}$ are pairwise almost disjoint, while $\mathscr{F}_{n}^{1}(\mu)$ is

$$
\left\{\mathbf{A} \in \mathscr{F}_{n}(\mu): \sum_{r=1}^{n} \mu\left(A_{r}\right)=1\right\} \text {. }
$$

Now let $P_{n}(\mu)$ denote the set of all $n$-tuples $\phi=\left(\phi_{1}, \phi_{2}, \ldots, \phi_{n}\right)$ such that $\phi_{r} \in L^{\infty}(\mu)$ 
and $\phi_{r} \geq 0$ a.e. $(\mu)$ for all $r$ and

$$
\sum_{r=1}^{n} \phi_{r}(\omega) \leq 1 \quad \text { a.e. }(\mu)
$$

Finally let $P_{n}^{1}(\mu)$ be the set of $\phi$ in $P_{n}(\mu)$ such that

$$
\sum_{r=1}^{n} \phi_{r}(\omega)=1 \quad \text { a.e. }(\mu) \text {. }
$$

(Note. We intend to regard $P_{n}(\mu)$ and $P_{n}^{1}(\mu)$ as subsets of $\left(L^{\infty}(\mu)\right)^{n}$. We permit ourselves the usual "abuse of language" in blurring the distinction between measurable functions and their equivalence classes.)

We shall be concerned with subsets of the product space $X^{n}$. We can make this into a Banach space by writing

$$
\left\|\left(x_{1}, x_{2}, \ldots, x_{n}\right)\right\|=\max _{1 \leq r \leq n}\left\|x_{r}\right\|_{\circ} .
$$

Then the dual space of $X^{n}$ is $X^{* n}$, the norm in the latter space being given by

$$
\left\|\left(x_{1}^{*}, x_{2}^{*}, \ldots, x_{n}^{*}\right)\right\|=\sum_{r=1}^{n}\left\|x_{r}^{*}\right\| .
$$

We can now state our first main theorem.

TheOREM 1.1. Let $m: \mathscr{F} \rightarrow X$ be a Liapounov measure. Then

and

$$
\left\{\left(m\left(A_{1}\right), m\left(A_{2}\right), \ldots, m\left(A_{n}\right)\right): \mathbf{A} \in \mathscr{F}_{n}(\mu)\right\}
$$

$$
\left\{\left(m\left(A_{1}\right), m\left(A_{2}\right), \ldots, m\left(A_{n}\right)\right): \mathbf{A} \in \mathscr{F}_{n}^{1}(\mu)\right\}
$$

are $\sigma\left(X^{n}, X^{* n}\right)$ - compact convex subsets of $X^{n}$.

This result will be established by showing that the two subsets of $X^{n}$ displayed in the statement are respectively equal to

and

$$
\left\{\left(m\left(\phi_{1}\right), m\left(\phi_{2}\right), \ldots, m\left(\phi_{n}\right)\right): \phi \in P_{n}(\mu)\right\}
$$

$$
\left\{\left(m\left(\phi_{1}\right), m\left(\phi_{2}\right), \ldots, m\left(\phi_{n}\right)\right): \phi \in P_{n}^{1}(\mu)\right\} .
$$

Since $P_{n}(\mu), P_{n}^{1}(\mu)$ are obviously convex sets, and are compact with respect to an appropriate topology (see $\$ 2$ ), it is then easy to conclude the proof. The proof of Theorem 1.1 will be concluded in $\$ 3$. In $\$ \S 4-6$ we shall establish results in the same spirit for infinite sequences $\left(A_{n}\right)$.

In addition to the references already given, we refer the reader, for some interesting comments on Liapounov's theorem and that of Dvoretzky-Wald-Wolfowitz, to [5]. (For further work on the subject of [5], though by methods not directly relevant to the present paper, see [12]). 
Finally it should be mentioned that Liapounov's theorem has been extended to von Neumann algebras by Azarnia and Wright [1]. It would be interesting to know whether Theorem 1.1, for instance, admits of a similar extension. Professor E. B. Davies has suggested that in looking for such generalizations one should seek to replace $L^{\infty}(\mu)$ not by a von Neumann algebra but by an appropriate ordered Banach space. This suggestion is not pursued in the present paper.

2. The compact convex sets $P_{n}(\mu)$ and $P_{n}^{1}(\mu)$. In this section the vector measure $m$ plays no part and $(\Omega, \mathscr{F}, \mu)$ can be taken to be an arbitrary probability space. The definitions of $P_{n}(\mu)$ and $P_{n}^{1}(\mu)$ have already been given in $\S 1$. They are easily seen to be compact convex subsets of the product space $\left(L^{\infty}(\mu)\right)^{n}$ when the latter carries the product weak $^{*}$ topology, i.e. the product topology constructed by starting with the topology $\sigma\left(L^{\infty}(\mu), L^{1}(\mu)\right)$ in each factor space. (Alternatively one may regard this topology on $\left(L^{\infty}(\mu)\right)^{n}$ as the weak* topology for $\left(L^{\infty}(\mu)\right)^{n} q u a$ dual of the Banach space $\left(L^{1}(\mu)\right)^{n}$. (One way to norm $\left(L^{1}(\mu)\right)^{n}$ so as to set up this duality has already been indicated in $\$ 1$. Another will be indicated in §7.) By the Krein-Milman theorem the sets $P_{n}(\mu)$ and $P_{n}^{1}(\mu)$ are the closed convex hulls of their sets of extreme points. In what follows we shall need to know what these extreme points are, and our next concern will therefore be to characterize them. Given any convex set $K$ we shall denote by $\partial_{e} K$ the set of all extreme points of $K$, and by a face of $K$ we shall mean any non-empty convex set $E \subseteq K$ such that if $x, y \in K$ and $0<t<1$ with $t x+(1-t) y \in E$ then $x, y \in E$. If $E$ is a face of $K$ then it is easy to see that $\partial_{e} E=E \cap \partial_{e} K$.

Proposition 2.1. The set $P_{n}^{1}(\mu)$ is a face of $P_{n}(\mu)$.

This is almost obvious. For if $\phi, \psi \in P_{n}(\mu)$ and $0<t<1$ with

$$
t \phi+(1-t) \psi \in P_{n}^{1}(\mu)
$$

then $\sum_{r=1}^{n} \phi_{r} \leq 1$ a.e., $\sum_{r=1}^{n} \psi_{r} \leq 1$ a.e. and so

$$
t \sum_{r=1}^{n} \phi_{r}+(1-t) \sum_{r=1}^{n} \psi_{r}=1 \quad \text { a.e. }
$$

Hence $\sum_{r=1}^{n} \phi_{r}=1$ a.e., $\sum_{r=1}^{n} \psi_{r}=1$ a.e. and so $\phi, \psi \in P_{n}^{1}(\mu)$.

This result with the remark immediately preceding it shows that it will be enough to characterize the points of $\partial_{e} P_{n}(\mu)$. Here is the characterization.

THEOREM 2.2. Let $\phi \in \partial_{e} P_{n}(\mu)$. Then there exists a member $\left(A_{1}, A_{2}, \ldots, A_{n}\right)$ of $\mathscr{F}_{n}(\mu)$ such that

$$
\phi=\left(1_{A_{1}}, 1_{A_{2}}, \ldots, 1_{A_{n}}\right) \text { a.e. }(\mu) .
$$

Conversely any $\phi$ constructed in this manner belongs to $\partial_{e} P_{n}(\mu)$. 
Here $1_{A_{1}}$, for instance, is the characteristic function of the set $A_{1}$. The symbol $\mathscr{F}_{n}(\mu)$ has already been defined in $\$ 1$. The basic idea for the proof is the same as that for Theorem 2 in [6], though the present situation is slightly more complicated.

To prove the theorem suppose that $\phi \in P_{n}(\mu)$ but that for no $A_{1} \in \mathscr{F}_{F}$ do we have $\phi_{1}=1_{A_{1}}$ a.e. $(\mu)$. Then for some $\varepsilon>0$ and $S \in \mathscr{F}$ we have $\mu(S)>0$ and $\varepsilon \leq \phi_{1} \leq 1-\varepsilon$ on $S$. Two cases now arise: (i) $\sum_{r=1}^{n} \phi_{r}=1$ a.e. in $S$, (ii) $\sum_{r=1}^{n} \phi_{r}<1$ on some subset $T$ of $S$ such that $T \in \mathscr{F}, \mu(T)>0$. (If $n=1$ then of course only case (ii) can occur, and we can take $T=S$.)

Consider case (i). We have $\varepsilon \leq \sum_{r=2}^{n} \phi_{r} \leq 1-\varepsilon$ a.e. in $S$. Writing, when $2 \leq r \leq n$,

$$
S_{r}=\left\{\omega \in S: \phi_{r}(\omega)>0\right\}
$$

we see that $S$ and $\bigcup_{r=2}^{n} S_{r}$ differ by at most a null set. Hence there exists an integer $k$ such that $2 \leq k \leq n$ and $\mu\left(S_{k}\right)>0$. Now since $\phi_{k}>0$ on $S_{k}$ there exist $\eta \in(0, \varepsilon]$ and $F \subseteq S_{k}$ such that $F \in \mathscr{F}, \mu(F)>0$ and $\phi_{k} \geq \eta$ on $F$. Now define, for $1 \leq i \leq n$,

$$
\phi_{i}^{ \pm}=\left\{\begin{array}{lll}
\phi_{i} & \text { if } & 1 \neq i \neq k \\
\phi_{1} \pm \eta 1_{F} & \text { if } & i=1, \\
\phi_{k} \mp \eta 1_{F} & \text { if } & i=k .
\end{array}\right.
$$

Then the two vector $\phi^{ \pm}$lie in $P_{n}(\mu)$ and $\phi=\frac{1}{2}\left(\phi^{+}+\phi^{-}\right)$. Since $\mu(F)>0$ this shows that $\phi$ is not extreme in $P_{n}(\mu)$.

Now consider case (ii). Choose $\delta \in(0, \varepsilon]$ and $H \in \mathscr{F}$ such that $\mu(H)>0, H \subseteq T$ and $\sum_{r=1}^{n} \phi_{r} \leq 1-\delta$ in $H$. Now define, for $1 \leq i \leq n$,

$$
\psi_{i}^{ \pm}=\left\{\begin{array}{lll}
\phi_{1} \pm \delta 1_{H} & \text { if } & i=1, \\
\phi_{i} & \text { if } & i \neq 1
\end{array}\right.
$$

Then $\psi^{ \pm} \in P_{n}(\mu)$ and $\phi=\frac{1}{2}\left(\psi^{+}+\psi^{-}\right)$. This tells us that $\phi$ is not extreme in $P_{n}(\mu)$.

It follows that if $\phi \in \partial_{e} P_{n}(\mu)$ then $\phi_{1}$ is equal a.e. to a characteristic function. But the same argument applies to each component $\phi_{r}$, and so we conclude that for each $r$ there exists $A_{r} \in \mathscr{F}$ such that $\phi_{r}=1_{A}$, a.e. Since $\phi \in P_{n}(\mu)$ it follows easily that $\left(A_{1}, A_{2}, \ldots, A_{n}\right) \in \mathscr{F}_{n}(\mu)$, so that $\phi$ is of the stated form. The converse statement is an easy exercise.

Corollary 2.3. Let $\phi \in \partial_{e} P_{n}^{1}(\mu)$. Then there exists a member $\left(A_{1}, A_{2}, \ldots, A_{n}\right)$ of $\mathscr{F}_{n}^{1}(\mu)$ such that

$$
\phi=\left(1_{A_{1}}, 1_{A_{2}}, \ldots, 1_{A_{n}}\right) \text { a.e. }(\mu) .
$$

Conversely, any $\phi$ constructed in this manner is a member of $\partial_{e} P_{n}^{1}(\mu)$. 
This follows from Theorem 2.2 and Proposition 2.1.

3. Proof of Theorem 1.1. In this section $\Omega, \mathscr{F}, X$ will have the same meanings as in $\S 1, m: \mathscr{F} \rightarrow X$ will be a vector measure, and $\mu$ will be a control measure for $m$. We shall interpret $X^{n}$ as a Banach space with dual $X^{* n}$, as already explained in $\$ 1$.

Given $\phi=\left(\phi_{1}, \phi_{2}, \ldots, \phi_{n}\right) \in\left(L^{\infty}(\mu)\right)^{n}$ we shall write

$$
m_{n}(\phi)=\left(m\left(\phi_{1}\right), m\left(\phi_{2}\right), \ldots, m\left(\phi_{n}\right)\right) \text {. }
$$

Given a subset $E$ of $\left(L^{\infty}(\mu)\right)^{n}$ we shall write $m_{n}[E]$ for the set $\left\{m_{n}(\phi): \phi \in E\right\}$.

Proposition 3.1. The map $m_{n}:\left(L^{\infty}(\mu)\right)^{n} \rightarrow X^{n}$ is continuous with respect to the product weak* topology in $\left(L^{\infty}(\mu)\right)^{n}$ and the weak topology $\sigma\left(X^{n}, X^{* n}\right)$ in $X^{n}$.

To see this is suffices to show that whenever $x_{1}^{*}, x_{2}^{*}, \ldots, x_{n}^{*}$ belong to $X^{*}$ the map

$$
\phi \rightarrow \sum_{r=1}^{n} x_{r}^{*} \circ m\left(\phi_{r}\right)
$$

is continuous with respect to the product weak* topology in $\left(L^{\infty}(\mu)\right)^{n}$. To prove that, let $f_{x^{*}}$, for each $x^{*} \in X^{*}$, be the density of the measure $x^{*} \circ m$ with respect to $\mu$ and recall that $f_{x^{*}} \in L^{1}(\mu)$. Then

$$
\sum_{r=1}^{n} x_{r}^{*} \circ m\left(\phi_{r}\right)=\sum_{r=1}^{n} \int \phi_{r} f_{x_{r}^{*}} d \mu,
$$

which makes the desired continuity property evident.

Corollary 3.2. The sets $m_{n}\left[P_{n}^{1}(\mu)\right]$ and $m_{n}\left[P_{n}(\mu)\right]$ are weakly compact convex subsets of $X^{n}$.

By the properties of $P_{n}^{1}(\mu)$ and $P_{n}(\mu)$ noted at the beginning of $\$ 2$ this follows at once from Proposition 3.1.

Now write

$$
N_{n}=\left\{\phi \in\left(L^{\infty}(\mu)\right)^{n}: m_{n}(\phi)=0\right\}
$$

Then $N_{n}$ is a linear subspace of $\left(L^{\infty}(\mu)\right)^{n}$ that is closed with respect to the product weak* topology.

LEMMA 3.3. If the measure $m$ is Liapounov then

(a) $P_{n}^{1}(\mu) \subseteq \partial_{e} P_{n}^{1}(\mu)+N_{n}$,

(b) $P_{n}(\mu) \subseteq \partial_{e} P_{n}(\mu)+N_{n}$.

The proof is similar to that for Theorem 2.2. The case $n=1$ of statement (b) is already well known (see [10], [8] and [9]).

We consider first statement (b). Let $\phi \in P_{n}(\mu)$ and let $\psi$ be an extreme point of $\left(\phi+N_{n}\right) \cap P_{n}(\mu)$. Suppose if possible that $\psi \notin \partial_{e} P_{n}(\mu)$. Then, by Theorem 2.2 , some component of $\psi, \psi_{1}$ say, is not a.e. equal to a characteristic function. But that means that 
for some $\varepsilon>0$ and $S \in \mathscr{F}$ with $\mu(S)>0$ we have $\varepsilon \leq \psi_{1} \leq 1-\varepsilon$ on $S$. We now distinguish two cases. Either (i) $\sum_{r=1}^{n} \psi_{r}=1$ a.e. on $S$ or (ii) $\sum_{r=1}^{n} \psi_{r}<1$ on some subset of $S$ that is of positive $\mu$-measure.

If we are in case (i) then, as in the proof of Theorem 2.2 we can find $\eta \in(0, \varepsilon], F$ a subset of $S$, and $k$ such that $F \in \mathscr{F}, \mu(F)>0,2 \leq k \leq n$, and $\psi_{k} \geq \eta$ on $F$. Now choose $\chi$ in $N(F)$ with $0<\|\chi\|_{\infty}<\eta$. For $1 \leq i \leq n$ let

$$
\psi_{i}^{ \pm}=\left\{\begin{array}{lll}
\psi_{i} & \text { if } & 1 \neq i \neq k, \\
\psi_{1} \pm \chi & \text { if } & i=1, \\
\psi_{k} \mp \chi & \text { if } & i=k .
\end{array}\right.
$$

Then $\psi^{ \pm} \in\left(\phi+N_{n}\right) \cap P_{n}(\mu)$ and $\psi=\frac{1}{2}\left(\psi^{+}+\psi^{-}\right)$. Hence $\psi$ is not extreme in $\left(\phi+N_{n}\right) \cap$ $P_{n}(\mu)$, contrary to hypothesis. To avoid the contradiction we must conclude that if $\psi$ is an extreme point of $\left(\phi+N_{n}\right) \cap P_{n}(\mu)$ then for some $A \subseteq S$ we have $\psi_{1}=1_{A}$ a.e. $(\mu)$. The same argument applies now to every component of $\psi$. Since $\psi \in P_{n}(\mu)$ it is immediate, by Theorem 2.2, that $\psi \in \partial_{e} P_{n}(\mu)$. Hence we have shown that $\phi \in \partial_{e} P_{n}(\mu)+N_{n}$.

Before examining case (ii) it is convenient to pause to note that formula (a) is now evident. For suppose that $\phi \in P_{n}^{1}(\mu)$ and that $\psi$ is an extreme point of $\left(\phi+N_{n}\right) \cap P_{n}^{1}(\mu)$. Then, by Proposition 2.1, $\psi$ is an extreme point of $\left(\phi+N_{n}\right) \cap P_{n}(\mu)$. By what we have already proved concerning statement (b) (note that we are now automatically in case (i)) we have $\psi \in \partial_{e} P_{n}(\mu)$. Since $\psi \in P_{n}^{1}(\mu)$ this shows that $\psi \in \partial_{e} P_{n}^{1}(\mu)$ (via Proposition 2.1). Hence $\phi \in \partial_{e} P_{n}^{1}(\mu)+N_{n}$, and the proof of formula (a) is complete.

We return to our consideration of formula (b). It remains for us to deal with case (ii). We assume therefore that for some $T \in \mathscr{F}$ we have $T \subseteq S, \mu(T)>0$ and $\sum_{r=1}^{n} \psi_{r}<1$ on $T$. As in the proof of Theorem 2.2 we choose $\delta \in(0, \varepsilon]$ and $H \in \mathscr{F}$ such that $\mu(H)>0$, $H \subseteq T$ and $\sum_{r=1}^{n} \psi_{r}<1-\delta$ on $H$. Now choose $\theta \in N(H)$ such that $0<\|\theta\|_{\infty}<\delta$, and, for $1 \leq i \leq n$, let

$$
\theta_{i}^{ \pm}=\left\{\begin{array}{lll}
\psi_{1} \pm \theta & \text { if } & i=1 \\
\psi_{i} & \text { if } & i \neq 1
\end{array}\right.
$$

Then $\boldsymbol{\theta}^{+}, \boldsymbol{\theta}^{-}$lie in $\left(\phi+N_{n}\right) \cap P_{n}(\mu)$ and $\psi=\frac{1}{2}\left(\theta^{+}+\theta^{-}\right)$. Hence $\psi$ is not extreme in $\left(\phi+N_{n}\right) \cap P_{n}(\mu)$, contrary to hypothesis. Arguing as before we conclude that $\psi \in$ $\partial_{e} P_{n}(\mu)$, and hence that $\phi \in \partial_{e} P_{n}(\mu)+N_{n}$. This disposes of case (ii) and completes the proof of formula (b), and of the Lemma.

THEOREM 3.4. Suppose that the measure $m$ is Liapounov. Then $m_{n}\left[\partial_{e} P_{n}^{1}(\mu)\right]=$ $m_{n}\left[P_{n}^{1}(\mu)\right]$ and $m_{n}\left[\partial_{e} P_{n}(\mu)\right]=m_{n}\left[P_{n}(\mu)\right]$. Consequently $m_{n}\left[\partial_{e} P_{n}^{1}(\mu)\right]$ and $m_{n}\left[\partial_{e} P_{n}(\mu)\right]$ are weakly compact convex subsets of $X^{n}$. 
For, by Lemma 3.3, we have

$$
m_{n}\left[P_{n}^{1}(\mu)\right] \subseteq m_{n}\left[\partial_{e} P_{n}^{1}(\mu)+N_{n}\right]=m_{n}\left[\partial_{e} P_{n}^{1}(\mu)\right] \subseteq m_{n}\left[P_{n}^{1}(\mu)\right]
$$

Hence $m_{n}\left[P_{n}^{1}(\mu)\right]=m_{n}\left[\partial_{e} P_{n}^{1}(\mu)\right]$. The same argument, mutatis mutandis, applies to $P_{n}(\mu)$. The rest is clear, by Corollary 3.2.

Corollary 3.5. (= Theorem 1.1). Suppose that the measure $m$ is Liapounov. Then

and

$$
\left\{\left(m\left(A_{1}\right), m\left(A_{2}\right), \ldots, m\left(A_{n}\right)\right): \mathbf{A} \in \mathscr{F}_{n}^{1}(\mu)\right\}
$$

$$
\left\{\left(m\left(A_{1}, m\left(A_{2}\right), \ldots, m\left(A_{n}\right)\right): \mathbf{A} \in \mathscr{F}_{n}(\mu)\right\}\right.
$$

are weakly compact convex subsets of $X^{n}$.

By the results of $\S 2$ this follows at once from Theorem 3.4.

4. Extension of Theorem 1.1 to infinite sequences $I$. We continue with the notation of $\S 3$. We shall denote by $\mathscr{F}_{\infty}(\mu)$ the set of all infinite sequences

$$
\mathbf{A}=\left(A_{1}, A_{2}, \ldots, A_{n}, \ldots\right)
$$

such that (i) $A_{n} \in \mathscr{F}$ for all $n$ and (ii) the $A_{n}$ are pairwise almost disjoint. Our object is to show that if $m: \mathscr{F} \rightarrow X$ is a Liapounov measure then

$$
\left\{\left(m\left(A_{1}\right), m\left(A_{2}\right), \ldots, m\left(A_{n}\right), \ldots\right): \mathbf{A} \in \mathscr{F}_{\infty}(\mu)\right\}
$$

is a convex subset of $X^{\infty}$, compact with respect to an appropriate topology. (By $X^{\infty}$ we of course mean the space of all infinite sequences $\left(x_{n}\right)_{n \geq 1}$ with $x_{n} \in X$ for all $n$ ).

It would seem natural to introduce at the same time (by analogy with $\mathscr{F}_{n}^{1}(\mu)$ ) the set

$$
\mathscr{F}_{\infty}^{1}(\mu)=\left\{\mathbf{A} \in \mathscr{F}_{\infty}(\mu): \sum_{n=1}^{\infty} \mu\left(A_{n}\right)=1\right\}
$$

and to prove a similar result about it. This we shall eventually do. However it turns out that it is not convenient to treat $\mathscr{F}_{\infty}(\mu)$ and $\mathscr{F}_{\infty}^{1}(\mu)$ together, and we shall deal in this section only with $\mathscr{F}_{\infty}(\mu)$. The method follows closely that for $\mathscr{F}_{n}(\mu)$, and we shall therefore be rather brief about it.

We shall denote by $\left(L^{\infty}(\mu)\right)^{\infty}$ the space of all infinite sequences

$$
\phi=\left(\phi_{1}, \phi_{2}, \ldots, \phi_{n}, \ldots\right)
$$

of members of $L^{\infty}(\mu)$. We take this space to be endowed with the product weak* topology. By $P_{\infty}(\mu)$ we understand the set

$$
\left\{\phi \in\left(L^{\infty}(\mu)_{+}\right)^{\infty}: \sum_{r=1}^{\infty} \phi_{r}(\omega) \leq 1 \quad \text { a.e. }(\mu)\right\} .
$$

It is easy to see (compare Dor [4]) that $P_{\infty}(\mu)$ is a compact convex subset of $\left(L^{\infty}(\mu)\right)^{\infty}$. (For the reader who would prefer to see $P_{\infty}(\mu)$ realized as a weak ${ }^{*}$-compact convex subset of a Banach space we shall indicate an alterntive treatment below). 
In the following theorem $m$ plays no role and $(\Omega, \mathscr{F}, \mu)$ can be an arbitrary probability space.

TheORem 4.1. Let $\phi \in \partial_{e} P_{\infty}(\mu)$. Then there exists an $\mathbf{A}=\left(A_{n}\right)$ in $\mathscr{F}_{\infty}(\mu)$ such that

$$
\boldsymbol{\phi}=\left(1_{A_{1}}, 1_{A_{2}}, \ldots, 1_{A_{n}}, \ldots\right) \text { a.e. }(\mu) .
$$

Conversely any $\phi$ constructed in this manner is an extreme point of $P_{\infty}(\mu)$.

This is proved by an obvious and easy modification of the demonstration of Theorem 2.2 and the details may safely be left to the reader.

For the rest of this section $\Omega, \mathscr{F}, X, m, \mu$ will be as in $\S 3$. We shall always suppose that $X^{\infty}$ carries the product weak topology (i.e. the product topology in which each factor space $X$ is given the topology $\left.\sigma\left(X, X^{*}\right)\right)$.

We define a map $m_{\infty}:\left(L^{\infty}(\mu)\right)^{\infty} \rightarrow X^{\infty}$ by

$$
m_{\infty}(\phi)=\left(m\left(\phi_{1}\right), m\left(\phi_{2}\right), \ldots, m\left(\phi_{n}\right) \ldots\right) .
$$

Proposition 4.2. The map $m_{\infty}:\left(L^{\infty}(\mu)\right)^{\infty} \rightarrow X^{\infty}$ is continuous with respect to the product weak ${ }^{*}$ topology in $\left(L^{\infty}(\mu)\right)^{\infty}$ and the product weak topology in $X^{\infty}$.

To see this it is enough to show that for each $r \geq 1$ and each $x^{*} \in X^{*}$ the map $\phi \mapsto x^{*} \circ m\left(\phi_{r}\right)$ is continuous with respect to the product weak* topology in $\left(L^{\infty}(\mu)\right)^{\infty}$. Since, as we have seen in $\$ 3$,

$$
x^{*} \circ m\left(\phi_{r}\right)=\int \phi_{r} f_{x^{*}} d \mu
$$

where $f_{x^{*}} \in L^{1}(\mu)$, this is clear.

Corollary 4.3. $m_{\infty}\left[P_{\infty}(\mu)\right]$ is a compact convex subset of $X^{\infty}$.

We can now conclude the business of the present section very rapidly by essentially the same argument as in \$3. The sequence of ideas is as follows. Let

$$
N_{\infty}=\left\{\phi \in\left(L^{\infty}(\mu)\right)^{\infty}: m_{\infty}(\phi)=0\right\} .
$$

LeMma 4.4. If the measure $m$ is Liapounov then $P_{\infty}(\mu) \subseteq \partial_{e} P_{\infty}(\mu)+N_{\infty}$.

THEorem 4.5. Suppose that the measure $m$ is Liapounov. Then $m_{\infty}\left[\partial_{e} P_{\infty}(\mu)\right]=$ $m_{\infty}\left[P_{\infty}(\mu)\right]$. Consequently $m_{\infty}\left[\partial_{e} P_{\infty}(\mu)\right]$ is a compact convex subset of $X^{\infty}$.

COROLlaRY 4.6. Suppose that the measure $m$ is Liapounov. Then the set of vectors

$$
\left\{\left(m\left(A_{1}\right), m\left(A_{2}\right), \ldots, m\left(A_{n}\right), \ldots\right): \mathbf{A} \in \mathscr{F}_{\infty}(\mu)\right\}
$$

is a compact convex subset of $X^{\infty}$.

Since the proofs of these results resemble closely those of the corresponding statements in $\S 3$, we omit them. 
We turn next to the problem of establishing similar results for $\mathscr{F}_{\infty}^{1}(\mu)$ and

$$
P_{\infty}^{1}(\mu)=\left\{\phi \in P_{\infty}(\mu): \sum \phi_{r}(\omega)=1 \quad \text { a.e. }(\mu)\right\} .
$$

Here it no longer suffices to make a simple adaptation of the argument of $\S 3$. The reason for this is that $P_{\infty}^{1}(\mu)$ is not a compact subset of $\left(L^{\infty}(\mu)\right)^{\infty}$. For if we write, for all $m, n \geq 0$, and all $\omega \in \Omega, \phi_{n}^{m}(\omega)=\delta_{m n}$ then

$$
\phi^{m} \in P_{\infty}^{1}(\mu) \text { and } \phi^{m} \rightarrow 0 \notin P_{\infty}^{1}(\mu)
$$

as $m \rightarrow \infty$. This means that new methods are required. What we shall do is to realize $P_{\infty}^{1}(\mu)$ as a weak* closed subset of the unit ball in a Banach dual space. By Alaoglu's theorem $P_{\infty}^{1}(\mu)$ will then be weak* compact, and by using the weak* topology we shall arrive at the appropriate analogues of the theorems of the present section.

5. A duality theorem. The purpose of this section is to realize $P_{\infty}^{1}(\mu)$ as a weak* compact convex subset of a Banach dual space. It will be convenient however to make a small change of notation, as follows. The space $\left(L^{\infty}(\mu)\right)^{\infty}$ will now be the set of all infinite sequences $\left(\phi_{n}\right)_{n \geq 0}$ of members of $L^{\infty}(\mu)$. By $P_{\infty}^{1}(\mu)$ we shall accordingly now mean the set

$$
\left\{\phi \in\left(L^{\infty}(\mu)_{+}\right)^{\infty}: \sum_{r=0}^{\infty} \phi_{r}(\omega)=1 \text { a.e. }(\mu)\right\} .
$$

We shall also assume that the definition of $P_{\infty}(\mu)$ is modified in the same way.

Now let $V$ be the Banach space $c$ of all real sequences $\xi=\left(\xi_{n}\right)_{n \geq 1}$ such that the finite limit $\xi_{0}:=\lim _{n \rightarrow \infty} \xi_{n}$ exists, with the norm given by $\|\xi\|=\sup _{n \geq 1}\left|\xi_{n}\right|$. The Banach dual of $c$ is the space $l^{1}$ of all absolutely summable real sequences $\eta=\left(\eta_{n}\right)_{n \geq 0}$ endowed with the norm $\|\mathfrak{\eta}\|=\sum_{n=0}^{\infty}\left|\eta_{n}\right|$, the duality being implemented by the pairing

$$
\langle\boldsymbol{\eta}, \xi\rangle=\sum_{n=0}^{\infty} \xi_{n} \eta_{n} .
$$

This is a duality of ordered Banach spaces. By $L_{v}^{1}(\mu)$ we shall understand the following space. Its members are the infinite sequences $\mathbf{f}=\left(f_{n}\right)_{n \geq 1}$ with (i) $f_{n} \in L^{1}(\mu)$ for all $n$, (ii) $\left(f_{n}(\omega)\right)_{n \geq 1} \in c$ for almost all $\omega \in \Omega$, and (iii)

$$
\|\mathbf{f}\|:=\int_{\Omega} \sup _{n \geq 1}\left|f_{n}(\omega)\right| \mu(d \omega)<\infty .
$$

It is easy to see that (with the obvious choice for positive cone) $L_{v}^{1}(\mu)$ is an ordered Banach space.

We have pointed out that $V^{*}$, the dual of $V$, is $l^{1}$. The space $L_{V}^{\infty} \cdot(\mu)$ is accordingly defined as follows. Its members are the infinite sequences $\phi=\left(\phi_{n}\right)_{n \geq 0}$ of members of 
$L^{\infty}(\mu)$ such that (i) $\left(\phi_{n}(\omega)\right)_{n \geq 0} \in l^{1}$ for almost all $\omega \in \Omega$, and (ii)

$$
\|\phi\|:=\underset{\omega \in \Omega}{\operatorname{ess} \sup } \sum_{n=0}^{\infty}\left|\phi_{n}(\omega)\right|<\infty .
$$

It is easy to show that $L_{V}^{\infty} \cdot(\mu)$ is an ordered Banach space.

We define a pairing of $L_{V}^{1}(\mu)$ and $L_{V^{*}}^{\infty}(\mu)$ as follows:

$$
\langle\boldsymbol{\phi}, \mathbf{f}\rangle=\int_{\Omega}\left(\sum_{n=0}^{\infty} f_{n}(\omega) \phi_{n}(\omega)\right) \mu(d \omega) .
$$

THEOREM 5.1. The ordered Banach space $L_{V}^{1}(\mu)$ has as dual the ordered Banach space $L_{V}^{\infty} \cdot(\mu)$, the duality being implemented by the above pairing. Moreover $P_{\infty}^{1}(\mu)$ is a weak ${ }^{*}$ compact convex subset of the latter space.

The duality statement in this theorem is a special case of a theorem about Bochner-integrable functions (see Theorem 1 in Ch. IV of [3]). However, it is much simpler to prove it directly, and this we now do.

Choose $\phi \in L_{V^{*}}^{\infty}(\mu)$ and write

$$
\Phi(f)=\langle\boldsymbol{\phi}, \mathbf{f}\rangle \quad\left(\mathbf{f} \in L_{V}^{1}(\mu)\right)
$$

We shall show that $\Phi \in\left(L_{V}^{1}(\mu)\right)^{*}$ and that $\|\Phi\|=\|\phi\|$. First we have, for all $\mathbf{f} \in L_{V}^{1}(\mu)$,

$$
\begin{aligned}
|\Phi(\mathbf{f})| & =\left|\int\left(\sum_{n=0}^{\infty} f_{n} \phi_{n}\right) d \mu\right| \leq \int \sum_{n=0}^{\infty}\left|f_{n} \phi_{n}\right| d \mu \\
& \leq \int_{m \geq 0} \sup \left|f_{m}\right| \sum_{n=0}^{\infty}\left|\phi_{n}\right| d \mu \leq\|\phi\| \int \sup _{m \geq 0}\left|f_{m}\right| d \mu \\
& =\|\boldsymbol{\phi}\|\|\mathbf{f}\|,
\end{aligned}
$$

which shows that $\|\Phi\| \leq\|\phi\|$. If $\phi$ is zero we clearly have equality here. To prove equality when $\phi$ is non-zero it suffices to show it for the case $\|\phi\|=1$. So suppose this, and let $\varepsilon>0$. Then we can find $g \in L^{1}(\mu)$ with $\|g\|_{1}=1, g \geq 0$ and

$$
\int g \sum_{n=0}^{\infty}\left|\phi_{n}\right| d \mu>1-\varepsilon
$$

Now define $f^{(n)} \in L_{V}^{1}(\mu)$ for each $n \geq 1$ as follows:

$$
f_{m}^{(n)}= \begin{cases}g \operatorname{sgn} \phi_{m} & (1 \leq m \leq n) \\ g \operatorname{sgn} \phi_{0} & (n<m)\end{cases}
$$

Then $\left\|f^{(n)}\right\| \leq 1$ and

$$
\begin{aligned}
\Phi\left(f^{(n)}\right) & =\int g \sum_{m=0}^{n}\left|\phi_{m}\right| d \mu+\int g \operatorname{sgn} \phi_{0} \sum_{m=n+1}^{\infty} \phi_{m} d \mu \\
& \leq\|\Phi\|\left\|f^{(n)}\right\| \leq\|\Phi\| .
\end{aligned}
$$


Letting $n \rightarrow \infty$ and using the dominated convergence theorem we deduce that

$$
1-\varepsilon<\int g \sum_{n=0}^{\infty}\left|\phi_{n}\right| d \mu \leq\|\Phi\|
$$

Since $\varepsilon$ was arbitrary and $\|\phi\|=1$ we deduce that $\|\phi\| \leq\|\Phi\|$. Hence we have shown that $\|\phi\|=\|\Phi\|$ for all $\phi \in L_{V^{*}}(\mu)$.

Next, we must show that all continuous linear functionals on $L_{V}^{1}(\mu)$ are of the type above. So let $\Psi: L_{V}^{1}(\mu) \rightarrow \mathbb{R}$ be a continuous linear functional and let $i_{r}: L^{1}(\mu) \rightarrow L_{V}^{1}(\mu)$ be defined by

$$
\left(i_{r} g\right)_{s}=\delta_{r s} g \quad\left(g \in L^{1}(\mu) ; r, s=1,2,3, \ldots\right) .
$$

Similarly let $j: L^{1}(\mu) \rightarrow L_{\nu}^{1}(\mu)$ be defined by

$$
(j g)_{s}=g \quad(s=1,2, \ldots) .
$$

Let us write $\Psi_{r}=\Psi \circ i_{r}, \Psi_{\infty}=\Psi \circ j$. Then $\Psi_{r}, \Psi_{\infty}$ belong to $\left(L^{1}(\mu)\right)^{*}$ and hence there exist $\psi_{r}, \psi_{\infty}$ in $L^{\infty}(\mu)$ such that

$$
\begin{aligned}
& \Psi_{r}(g)=\int g \psi_{r} d \mu \quad(r=1,2, \ldots) \\
& \Psi_{\infty}(g)=\int g \psi_{\infty} d \mu .
\end{aligned}
$$

Now take an arbitrary $\mathbf{f}$ in $L_{V}^{1}(\mu)$. Then we can write

$$
\mathbf{f}=j f_{0}+\sum_{r=1}^{\infty} i_{r}\left(f_{r}-f_{0}\right)
$$

where the series on the right converges in norm. Hence we have

$$
\Psi(\mathbf{f})=\int f_{0} \psi_{\infty} d \mu+\sum_{r=1}^{\infty} \int\left(f_{r}-f_{0}\right) \psi_{r} d \mu .
$$

We now want to show that $\sum_{r=1}^{\infty}\left|\psi_{r}\right|$ belongs to $L^{\infty}(\mu)$. To see this, let $g \in L^{1}(\mu)$ with $\|g\|_{1} \leq 1$ and $g \geq 0$, and let $\mathbf{f}^{(n)} \in L_{V}^{1}(\mu)$ be defined, for $n=1,2, \ldots$, by

$$
f_{m}^{(n)}= \begin{cases}g \operatorname{sgn} \psi_{m} & (1 \leq m \leq n), \\ 0 & (n<m) .\end{cases}
$$

Then $\mathbf{f}^{(n)} \in L_{V}^{1}(\mu),\left\|\mathbf{f}^{(n)}\right\| \leq 1$, and

$$
\Psi\left(\mathbf{f}^{(n)}\right)=\int g \sum_{r=1}^{n}\left|\psi_{r}\right| d \mu .
$$


On the other hand

$$
\Psi\left(\mathbf{f}^{(n)}\right) \leq\|\Psi\|\left\|\mathbf{f}^{(n)}\right\| \leq\|\Psi\|
$$

By the monotone convergence theorem we therefore deduce that $g \sum_{r=1}^{\infty}\left|\psi_{r}\right| \in L^{1}(\mu)$ and that

$$
\int g \sum_{r=1}^{\infty}\left|\psi_{r}\right| d \mu \leq\|\Psi\|
$$

whenever $g$ satisfies the conditions stipulated above. It follows that $\sum_{r=1}^{\infty}\left|\psi_{r}\right|$ belongs to $L^{\infty}(\mu)$ with

$$
\operatorname{ess} \sup \sum_{r=1}^{\infty}\left|\psi_{r}\right| \leq\|\Psi\| .
$$

(In the foregoing discussion $\sum_{r=1}^{\infty}$ means, as is customary, $\sum_{1 \leq r<\infty}$, and not $\sum_{1 \leq r \leq \infty}$. )

Now let $\psi_{0}=\psi_{\infty}-\sum_{r=1}^{\infty} \psi_{r}$ and let

$$
\psi=\left(\psi_{0}, \psi_{1}, \psi_{2}, \ldots\right)
$$

Then $\psi \in L_{V^{*}}^{\infty}(\mu)$ and $\Psi(\mathbf{f})=\langle\boldsymbol{\psi}, \mathbf{f}\rangle$ for all $\mathbf{f} \in L_{V}^{1}(\mu)$.

Next we consider the question of order. Suppose that $\phi \in L_{V}^{\infty}(\mu)$. We must show that $\phi \geq 0$ if and only if $\langle\phi, \mathbf{f}\rangle \geq 0$ for all $\mathbf{f}$ satisfying $0 \leq \mathbf{f} \in L_{V}^{1}(\mu)$. The necessity is clear. To prove the sufficiency we consider a number of special cases. Suppose then that $\langle\boldsymbol{\phi}, \mathbf{f}\rangle \geqslant 0$ whenever $0 \leq \mathbf{f} \in L_{V}^{1}(\mu)$. If all coordinates of $\mathbf{f}$ are zero save the $r$ th we get

$$
\langle\boldsymbol{\phi}, \mathbf{f}\rangle=\int_{\Omega} \phi_{r} f_{r} d \mu
$$

This shows that $\phi_{r} \geq 0$ for all $r \geq 1$. It remains to prove that $\phi_{0} \geq 0$. To see this suppose that $0 \leq h \in L^{\prime}(\mu)$ and let, for all $m, n \geq 1$,

$$
h_{n}^{(m)}= \begin{cases}h & (n \geqslant m), \\ 0 & (n<m) .\end{cases}
$$

Then $\mathbf{h}^{(m)} \in L_{V}^{1}(\mu)$ and $h \geq 0$, so that

$$
0 \leq\left\langle\boldsymbol{\phi}, \mathbf{h}^{(m)}\right\rangle=\int \phi_{0} h d \mu+\int \sum_{r=m}^{\infty} \phi_{r} h d \mu .
$$

Letting $m \rightarrow \infty$ we deduce, by the dominated convergence theorem, that $\int \phi_{0} h d \mu \geq 0$. Hence $\phi_{0} \geq 0$; and so we have proved that $\phi \geq 0$, as desired. This completes the discussion of duality, and it remains to deal with the statement about $P_{\infty}^{1}(\mu)$. 
Since $P_{\infty}^{1}(\mu)$ is a subset of the unit ball in $L_{V}^{\infty}(\mu)$ it suffices for the weak*compactness assertion to prove that $P_{\infty}^{1}(\mu)$ is a weak ${ }^{*}$ closed subset of the unit ball. Now $P_{\infty}(\mu)$ is the intersection of the (weak* closed) positive cone in $L_{V^{*}}^{\infty}(\mu)$ with the unit ball, and hence is weak* compact. But if $\phi \in P_{\infty}(\mu)$ then $\phi \in P_{\infty}^{1}(\mu)$ if and only if

$$
\langle\phi, j g\rangle=\mu(g) \quad\left(g \in L^{1}(\mu)\right) .
$$

So if we write, for each $g \in L^{1}(\mu)$,

$$
H_{g}=\left\{\phi \in L_{V}^{\infty} \cdot(\mu):\langle\phi, j g\rangle=\mu(g)\right\}
$$

then

$$
P_{\infty}^{1}(\mu)=P_{\infty}(\mu) \cap \cap\left\{H_{g}: g \in L^{1}(\mu)\right\} .
$$

Since each $H_{g}$ is weak* closed this makes it clear that $P_{\infty}^{1}(\mu)$ is weak* compact. This completes the proof of Theorem 5.1.

We record for future use a characterization of $\partial_{e} P^{1}(\mu)$.

TheOREM 5.2. Let $\phi \in \partial_{e} P_{\infty}^{1}(\mu)$. Then there exists an $\mathbf{A} \in \mathscr{F}_{\infty}^{1}(\mu)$ such that

$$
\phi=\left(1_{A_{0}}, 1_{A_{1}}, \ldots, 1_{A_{n}}, \ldots\right) \text { a.e. }(\mu) .
$$

Conversely, any $\phi$ constructed in this manner is an extreme point of $P_{\infty}^{1}(\mu)$.

This is proved by an easy modification of the argument for Theorem 2.2.

6. Extension of Theorem 1.1 to infinite sequences II. We take $\Omega, \mathscr{F}, X, m, \mu$ to be as in $\$ 2$. We shall also use the notation and results of $\$ 5$. By us $(X)$ we shall understand the space of all unconditionally summable sequences $\mathbf{x}=\left(x_{n}\right)_{n \geq 0}$ in $X$. This is a real vector space and we topologize it as follows. For each $x^{*} \in X^{*}$ and each $\xi \in c$ construct the map

$$
\mathbf{x} \mapsto \sum_{n=0}^{\infty} \xi_{n} x^{*}\left(x_{n}\right) .
$$

We define $\tau$ to be the topology in $u s(X)$ that makes all these maps continuous. If $\phi \in L_{V^{*}}^{\infty}(\mu)$ then it is easy to see, by the dominated convergence theorem for integrals with respect to a vector measure, that

$$
m_{\infty}(\phi):=\left(m\left(\phi_{0}\right), m\left(\phi_{1}\right), \ldots\right)
$$

belongs to $u s(X)$. (Note that the symbol $m_{\infty}$ is now used in a sense consistent with the notational changes made at the start of $\$ 5$ ).

THEOREM 6.1. The map $m_{\infty}: L_{V^{*}}^{\infty}(\mu) \rightarrow u s(X)$ is continuous with respect to the topologies $\sigma\left(L_{V}^{\infty} \cdot(\mu), L_{V}^{1}(\mu)\right.$ and $\tau$.

To see this we have merely to check that if $\xi \in c$ and $x^{*} \in X^{*}$ then the map

$$
\phi \mapsto \sum_{n=0}^{\infty} \xi_{n} x^{*} \circ m\left(\phi_{n}\right)
$$


from $L_{V^{*}}^{\infty}(\mu)$ to $\mathbb{R}$ is continuous with respect to $\sigma\left(L_{V}^{\infty}(\mu), L_{V}^{1}(\mu)\right)$. However, this fact is evident from the equation

$$
\sum_{n=0}^{\infty} \xi_{n} x^{*} \circ m\left(\phi_{n}\right)=\sum_{n=0}^{\infty} \int \xi_{n} \phi_{n} d\left(x^{*} \circ m\right)=\int \sum_{n=0}^{\infty} \xi_{n} \phi_{n} f_{x^{*}} d \mu
$$

and the fact that $\left(\xi_{n} f_{x^{*}}\right)_{n \geq 1}$ belongs to $L_{V}^{1}(\mu)$.

Recalling Theorem 5.1 we now have the following result.

Corollary 6.2. $m_{\infty}\left[P_{\infty}^{1}(\mu)\right]$ is a $\tau$-compact subset of us $(X)$.

We can now complete the business of the present section very rapidly by means of the argument, mutatis mutandis, used in $\$ 3$. We now use the symbol $N_{\infty}$ in the following sense:

$$
N_{\infty}=\left\{\phi \in L_{V^{*}}^{\infty}(\mu): m_{\infty}(\phi)=0\right\} .
$$

As in $\$ 4$ we now have the following sequence of results.

LemMa 6.3. If the measure $m$ is Liapounov then

$$
P_{\infty}^{1}(\mu) \subseteq \partial_{e} P_{\infty}^{1}(\mu)+N_{\infty} .
$$

THEOREM 6.4. Suppose that the measure $m$ is Liapounov. Then $m_{\infty}\left[\partial_{e} P_{\infty}^{1}(\mu)\right]=$ $m_{\infty}\left[P_{\infty}^{1}(\mu)\right]$. Consequently $m_{\infty}\left[\partial_{e} P_{\infty}(\mu)\right]$ is a $\tau$-compact convex subset of $u s(X)$.

Corollary 6.5. Suppose that the measure $m$ is Liapounov. Then the set of vectors

$$
\left\{\left(m\left(A_{0}\right), m\left(A_{1}\right), \ldots\right): \mathbf{A} \in \mathscr{F}_{\infty}^{1}(\mu)\right\}
$$

is a $\tau$-compact subset of us $(X)$.

7. Concluding remarks. In $\$ 5$ and $\$ 6$ we have shown how to prove certain theorems in the Dvoretsky-Wald-Wolfowitz spirit via a particular realization of $P_{\infty}(\mu)$ and $P_{\infty}^{1}(\mu)$ as weak* compact subsets of a Banach dual space. It is worth pointing out that this approach could have been adopted throughout the present paper.

For example in $\$ 2$ we could have proceeded as follows. Let $V$ denote the space $l_{n}^{\infty}$, $V^{*}$ its dual $l_{n}^{1}$. Then $L_{V}^{1}(\mu)$ will be the space of all $n$-tuples $\mathbf{f}=\left(f_{1}, f_{2}, \ldots, f_{n}\right)$ with $f_{r} \in L^{1}(\mu)$ for all $r$ and

$$
\|\mathbf{f}\|=\int_{1 \leq r \leq n}\left|f_{r}\right| d \mu,
$$

while $L_{V^{*}}^{\infty}(\mu)$ now consists of all $n$-tuples $\phi=\left(\phi_{1}, \phi_{2}, \ldots, \phi_{n}\right)$ with $\phi_{r} \in L^{\infty}(\mu)$ for all $r$ and

$$
\|\phi\|=\operatorname{ess} \sup \sum_{r=1}^{n}\left|\phi_{r}\right|
$$

Then $L_{V}^{1}(\mu)$ and $L_{V^{*}}^{\infty}(\mu)$ are ordered Banach spaces, the second being the dual of the 
first. Moreover $P_{n}(\mu)$ and $P_{n}^{1}(\mu)$ are weak*-compact convex subsets of $L_{V^{*}}^{\infty}(\mu)$. And $\S 3$ can be rewritten from this point of view (the only change required being to work with $L_{V}^{\infty} \cdot(\mu)$ with its weak* topology throughout and to rewrite Proposition 3.1 and its proof accordingly). All this should be immediately evident for the reader who has worked through $\$ \S 5$ and 6 . This approach is more complicated than that actually adopted in $\S \S 2$ and 3 , but also somewhat more natural, since $P_{n}(\mu)$ is then precisely the positive part of the unit ball in $L_{V^{*}}(\mu)$.

$\$ 4$ can also be rewritten in the same spirit. Here we must take $V=c$ and $V^{*}=l^{\infty}$ and follow the reasoning of $\$ \$ 5$ and 6 fairly closely.

In view of what has been said the details of the above modifications may be left as an exercise.

\section{REFERENCES}

1. N. Azarnia and J. D. M. Wright, On the Lyapunov-Knowles theorem, Quart. J. Math. Oxford Ser. 2, 33 (1982), 257-261.

2. R. G. Bartle, N. Dunford and J. T. Schwartz, Weak compactness and vector measures, Canad. J. Math. 7 (1955), 289-305. 1977).

3. J. Diestel and J. J. Uhl Jr., Vector measures, AMS Math. Surveys No. 15 (Providence R.I.

4. L. E. Dor, On projections in $L_{1}$, Ann. of Math. 102 (1975), 463-474.

5. L. E. Dubins and E. H. Spanier, How to cut a cake fairly, Amer. Math. Monthly 68 (1961), 1-17.

6. A. Dvoretsky, A. Wald and J. Wolfowitz, Relations among certain ranges of vector measures, Pacific J. Math 1 (1951), 51-74.

7. A. Dvoretsky, A. Wald and J. Wolfowitz, Elimination of randomization in certain statistical decision procedures and zero-sum two-person games, Ann. Math. Statist. 22 (1951), 1-21.

8. J. F. C. Kingman and A. P. Robertson, On a theorem of Liapounov, J. London. Math. Soc. 43 (1968), 347-351.

9. G. Knowles, Liapounov vector measures, SIAM J. Control 13 (1974), 294-303.

10. J. Lindenstrauss, A short proof of Liapounov's convexity theorem, J. Math. Mech. 15 (1966), 971-972.

11. A. Lyapunov, Sur les fonctions-vecteurs complètement additives, Izv. Akad. Nauk SSSR Ser. Mat. 4 (1940), 465-478.

12. D. R. Woodall, Dividing a cake fairly, J. Math. Anal. Appl. 78 (1980), 233-247.

Mathematical Institute

24-29 ST GiLes

OXFORD OX1 3LB. 